\title{
Zur Debatte um den «Sonderweg deutsche Medienwissenschaft>
}

\author{
Besprochen von CLAUDIA BREGER (Indiana University, Bloomington)
}

Auf den ersten Blick scheint kaum etwas unzeitgemäßer als eine deutsche Sonderwegsdebatte im Jahre 2008/2009. Ich erinnere mich, dass die entsprechende Kontroverse in den Geschichtswissenschaften aktuell war, als ich im Westberlin der späten achtziger Jahre Proseminare zu belegen begann. ${ }^{1}$ Seitdem ist methodologisch viel passiert, und aktuelle historiografische Paradigmen akzentuieren die Bedeutung des Transnationalen ${ }^{2}-$ d. h. die Notwendigkeit, die Geschichte(n) auch Europas im Hinblick auf intellektuell-produktive ebenso wie gewaltsame Austauschprozesse zu schreiben, zwischen Sklavenhandel, Exil und Immigration, Kolonialismus und Globalisierung. Sollten sich die Fragestellungen, die der hier zu kommentierenden Kontroverse zugrunde liegen, demgegenüber auf das Niveau von Feuilletongeraune und Anzeigenkampagne begeben, auf dem die Interpellation ins Nationale (‘Du bist Deutschland!)) in den letzten Jahren allemal wieder Konjunktur gehabt hat?

Selbstverständlich nicht. Die Mehrzahl der Debattenbeiträge, allen voran Geoffrey Winthrop-Youngs eröffnende Provokation, sind methodologisch gerade den kritisch-analytischen Blicken auf nationale Identitäten verpflichtet, die seit den 198oern im Zeichen des Postkolonialismus, der Globalisierung und nun eben der Transnationalität entwickelt worden sind - oder, näher am Stein des Anstoßes formuliert: im Zeichen der (nicht mit 〈Kulturwissenschaft〉 zu verwechselnden) Cultural Studies, die, wie Winthrop-Young formuliert, "beispielsweise in England" jene «akademische Energie auf sich gezogen" haben, die in Deutschland der «Medienerforschung" gegolten hat (S.121). Nationen sind diesen Paradigmen zufolge (ähnlich wie, worauf gleich einleitend insistiert sei, akademische Disziplinen) medial erzeugte Imagined Communities $^{3}$, die konstitutiv instabil, heterogen und von Kämpfen um soziale Hegemonie durchzogen sind und deren Historizität und Prozesscharakter die Möglichkeit ihrer kritischen Reartikulation einschließen. In vieler Hinsicht sind es überhaupt erst transnationale Austauschprozesse, die Nationen ihre Konturen verleihen - und zwar nicht nur in der offensichtlich von Sklavenhandel und Kolonialismus geprägten «Neuen Welt», sondern auch im preußisch-deutschen Reich, das sich angesichts einwanderungswilliger $\mathrm{Hu}$ genotten großzügig aufgeklärte Toleranz auf seine Fahnen schrieb, später allerdings aus Angst davor, dass «Kolonialsubjekte> Rechte auf Staatsbürgerschaft einklagen könnten, letztere mit Gültigkeit für den Rest des 20. Jahrhunderts an das Kriterium des (implizit: weißen) Blutes knüpfte. ${ }^{4}$

Auch die deutschen Medienwissenschaften sind, wie Winthrop-Youngs (im Rekurs vor allem auf McLuhan) kkanadisches Reflexionen und auf dem Siegener Podium explizit Erhard Schüttpelz festhielten, das Produkt solch transnationaler Austauschprozesse. Als Friedrich Kittler, dessen Werk in Nordamerika allzu oft für die deutsche Medienwissenschaft insgesamt einstehen muss, antrat, den Geist (wennschon, wie er heute unterstreicht, primär im Singular) aus den Geisteswissenschaften auszutreiben, bezog er sich theoretisch bekanntlich nicht nur auf die Kanadier, sondern auch auf den in Deutschland tatsächlich oft unter nationalem label verhandelten französischen Poststrukturalismus. Versetzt mit der Freiburger Provinz Heidegger'scher Seinsbegriffe ergab das allenfalls national ‘hybride` Theorie. Die Frage ist allerdings, ob, bzw. in welcher Hinsicht, solche 'gemischten» - und für das Fach insgesamt vielfältig transnationalen - intellektuellen Genealogien die Sonderwegsfrage, wie auf dem Siegener Podium ebenfalls diskutiert wurde, schon hinfällig machen. Gerade wenn man die methodologische Einsicht in die grundsätzlich transnationale Konstitution der Nation ernst nimmt, gewinnt die Untersuchung der sie erzeugenden Prozesse mehr oder minder kollektiver Identifizierung, diskursiver Verdichtung und institutioneller Kristallisierung ja von hier erst ihre Konturen. Im Hinblick auf die Medienwissenschaften ginge es dann um Rezeptionsakzente, um die lokale Verstärkung, Umlenkung oder Verknüpfung spezifischer Theorieströme und - wie auch in Siegen wiederholt formuliert - die Verschränkung von Theorieproduktion mit spezifischen institutionellen Bedingungen. 
Meine eigene Antwort auf die Debattenfrage beginnt nichtsdestotrotz mit dem Plädoyer, im vorliegenden Kontext auf den historisch allzu eindeutig, dramatisch und methodologisch reduktiv besetzten Begriff des Sonderwegs zu verzichten. Auch über die Ebene des labeling hinaus bleibt im Einzelnen nachzufragen, wofür es jeweils sinnvoll ist, die Frage nach aktuellen intellektuell-akademischen Konfigurationen im Rekurs auf die Kategorie der Nation zu stellen. Diese Differenzierungsarbeit lässt sich allerdings genau im Ernstnehmen der - sich überlappenden, aber in ihren Implikationen auch divergenten - Thesen leisten, die in der Zeitschrift für Kulturwissenschaften und in Siegen im Einzelnen vorgebracht wurden.

In einer Geste spielerischer Mimikry an McLuhans globalgeschichtliche «Brachialität» schlägt Winthrop-Young vor, dass «Produktivität und Profil der deutschen Medientheorie» als der «akademische Niederschlag einschneidender [...] kollektiver Erfahrungen" zu verstehen seien, d.h. genauer, dass sie sich der geteilten Wahrnehmung von überwältigender Medienmacht zwischen Modernisierung und Goebbel'scher Propagandamaschine verdanken (S.122, 124). Im Gegensatz zum Interesse der anglo-amerikanischen Cultural Studies an sozialen Binnendifferenzen wie race und gender haben hierzulande solche Varianten z. B. von Systemtheorie, Poststrukturalismus und Konstruktivismus Konjunktur gehabt, die - im Stil «inkompatibler Supertheorien» mit «konzeptuellen Ausschließlichkeitsansprüchen» - Homogenisierungszwänge und die Verselbständigung der Medien, eher denn die potentiell kritischen Spielräume individueller NutzerInnen, akzentuieren (S. 118, 122).

Motivisch korrespondiert diese Diagnose sowohl mit den Winthrop-Youngs Provokation insgesamt unterstützenden - Antworten Rüdiger Campes und Helmut Lethens ${ }^{5}$ als auch mit Gumbrechts Eröffnungsreferat zur Siegener Podiumsdiskussion. Gumbrecht brachte (in meiner leichten Revision seiner Dezimalordnung) vier epistemologisch signifikante Momente deutscher geisteswissenschaftlicher Diskurse ins Spiel: 1. die "Sehnsucht nach dem Referenten", 2. einen nicht primär marxistischen Materialismus, 3. die (in Kittlers Verabschiedung der Software kulminierende) Dekonstruktion des Subjekts und 4. die Dominanz welt- und zeitumspannender Entwürfe von der Geschichtsphilosophie bis zur philosophischen Anthropologie des 20. Jahrhunderts. ${ }^{6}$

Vielleicht ist, wie ich zu bedenken geben würde, der Einfluss letzterer selbst in Winthrop-Youngs Reformulierungen von McLuhans "globale[r] Mediengrammatik» (S.148) noch zu spüren (deren Sonderwegsimplikationen, wie Friedrich Balke unterstreicht, bereits von Helmuth Plessner vorgebracht worden waren): Die Rede von kollektiven Medienerfahrungen, bleibt mit ihrer unterstellten Allgemeinverbindlichkeit und ihrem Akzent aufWahrnehmung (eher denn z. B.

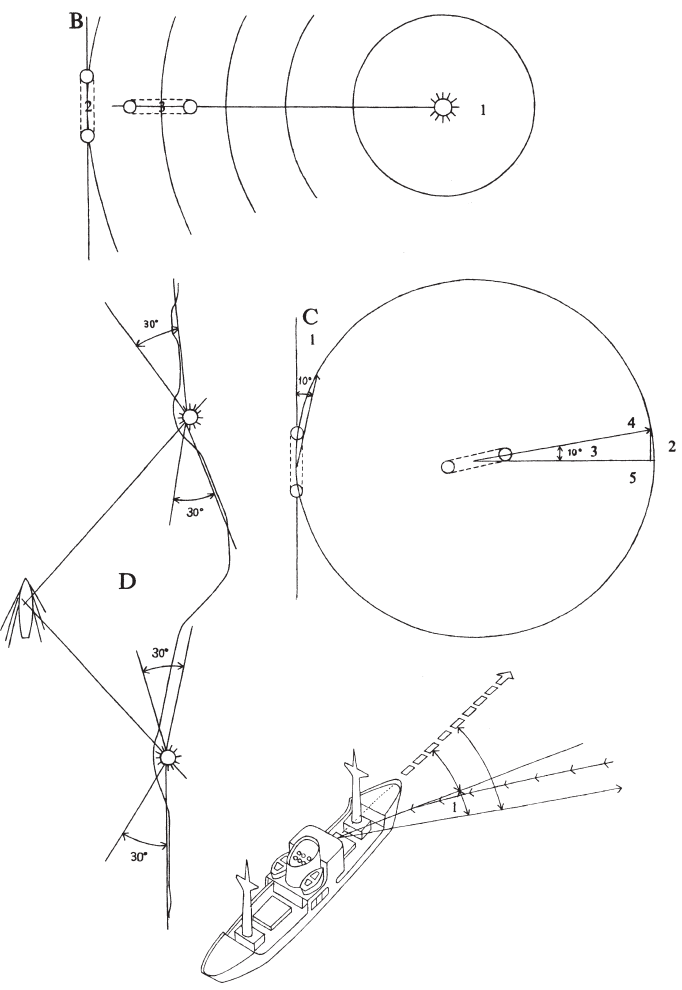

Ideologie) den diagnostizierten deutschen ‘Diskursphänomenen` immanent. Unmissverständlicher scheint mir der von Winthrop-Young in anderem Zusammenhang ebenfalls gebrauchte Begriff der Hegemonie zu sein, der die Prominenz bestimmter Themen und Theoreme im Spiel sozio-symbolischer Mächte bezeichnen, dabei vor allem in der Verbindung mit einem Adjektiv wie relativ aber zugleich unterstreichen kann, dass ein genauerer Blick auf die Vielfältigkeit der deutschen Theorielandschaft eben auch alternative Zugänge zu sehen gibt.

Als Aussage über relativ hegemoniale Theoriemotive verdienen die kombinierten Diagnosen meines Erachtens allemal eine ausführlichere Diskussion. Allerdings ist dabei - um noch eine Verschiebung vorzuschlagen - der Blick vielleicht weniger auf die Medienwissenschaft als solche zu richten, die an verschiedenen deutschen Universitäten methodologisch und curricular ganz unterschiedlich profiliert ist (wie sich während der Podiumsdiskussion in der lokalpatriotischen Appropriation des Sonderwegmotivs durch die Siegener Gastgeber andeutete), als auf die - u. a. dezidiert medientheoretisch profilierte - deutsche Kulturwissenschaft im Sinne eines institutionell-disziplinär primär an der HumboldtUniversität zu Berlin verankerten, aber auch darüber hinaus einflussreichen theoretischen Konkurrenzmodells zu den Cultural Studies. 
Als methodologisches Paradigma eher denn Gegenstand ist das Mediale hier, wie Florian Cramer in seinem Beitrag zur (mittlerweile auch online fortgeführten) Debatte ausgeführt hat $^{7}$, zum Fokus einer an der Warburg-Schule orientierten, interdisziplinären geisteswissenschaftlichen Untersuchung von Wissensgeschichte und kulturellem Gedächtnis geworden. Im politischen Klima des vereinigten Deutschlands bzw., wie im Hinblick auf die Kittler-Gumbrecht-Allianz auf dem Siegener Podium geopolitisch zu generalisieren wäre, auch in der Abwendung der 1968er von ihrer politischen Vergangenheit - hat diese deutsche Kulturwissenschaft sich tatsächlich nicht zuletzt gegen die sozio-symbolischen Egalisierungsprojekte der anglo-amerikanischen Cultural Studies konstituiert. Von letzteren beeinflusste alternative Strömungen sammelten sich, wie Cramer ausführt, demgegenüber in Deutschland zunächst primär im Medienkunstbereich (um Zeitschriften wie Texte zur Kunst herum), obgleich sie von dort, wie ich ergänzen würde, mittlerweile auch auf Mittelbaustellen und in den letzten Jahren auf neuberufene Professuren gelangt sind.

Auch das so zugespitzt umrissene Paradigma deutscher Kulturwissenschaft selbst ist kein monolithischer Theorienblock, sondern eher ein von alternativen Fäden durchwirktes, breitmaschiges Motivgewebe. Allerdings fällt auch mir - als einer akademisch in Deutschland sozialisierten, aber selbst transatlantisch migrierten Leserin aktueller deutschsprachiger Theorie - beispielsweise die Prominenz von Heteronomie-Motiven auf, die eben nicht nur in der Kittler-Schule zu beobachten ist, sondern z. B. auch in der Medientheorie (denominativ philosophischer Herkunft) von Sybille Krämer, die von Winthrop-Young (zu recht) als «intellektuell ... bestechend [e] Alternative" gepriesen wird (S.120). So ist der Bote, in dem das Mediale in Krämers jüngster Monografie figuralisiert wird, maßgeblich durch seine Heteronomie nicht bloß in dem Sinne bestimmt, dass er nicht souverän ist (was auch ich jederzeit unterschreiben würde), sondern in dem (stärkeren, mit Handlungsfähigkeit kaum noch kompatibel erscheinenden) einer nicht-dialogischen, von Krämer primär in religiösen und monarchischen Bildern beschriebenen "Fremdbestimmung». ${ }^{8}$

Einem Heteronomiemotiv verpflichtet ist auch Gumbrechts Insistenz darauf, die von ihm diagnostizierte Sonderentwicklung nicht bewerten, sondern schlicht beschreiben zu wollen, da sie ohnehin nicht ohne weiteres umkehrbar und ihre Evaluation insofern ohne politisch-pragmatische Konsequenz sei. Auf dem Podium hat ihm Schüttpelz diesbezüglich explizit widersprochen, und ich kann mir den ergänzenden Kommentar nicht verkneifen, dass es genau die hier von Gumbrecht wieder verwendete Rhetorik neutraler Beschreibung war, die einst die von ihm selbst retrospektiv in Gießen als «Neohermeneutik» beschriebene Hermeneutik- kritik des Dekonstruktivismus trug. In anderen Worten: Die Frage, wie die in der Debatte konturierten Befunde zur relativen Hegemonie spezifischer Theoriemotive zu bewerten, und wie ihnen zu begegnen ist, ist allemal entscheidend.

Im Prinzip dürfte unter allen DebattantInnen Konsens sein, dass es nicht darum gehen kann, die Welt im 21. Jahrhundert am deutschen Theoriewesen - oder, mit der von Gumbrecht und Kittler auf dem Podium unterstrichenen Heideggervokabel, Sein - noch einmal genesen zu lassen. ${ }^{9}$ Ebensowenig sollte man den Verweis eines kalifornischen Gastes auf die Stärke deutscher Universitäten oder seine Rhetorik des Anti-Anti-Nationalen, und schon gar nicht das auf dem Podium anschließend laut gewordene Plädoyer für mehr Export deutscher Medientheorie (Lovink, vorsichtiger auch Winkler) mit der eingangs anzitierten neuen Konjunktur des Nationalen im populären Diskurs kurzschließen. Bei Lovink und Winkler wenigstens geht es dezidiert um die politischen Reflexionspotentiale, welche die in der deutschen Theorie produktiv akzentuierte Vermittlung - so Winklers Vorschlag - zwischen dem Abstrakten und dem Konkreten freisetzen kann. In Abgrenzung von amerikanischem Pragmatismus würde ich das, anders als Winkler, allerdings nicht so pauschal formulieren: Zu unterscheiden wäre hier z. B. zwischen (in der Tat hegemonial (pragmatischen`) amerikanischen Philosophietraditionen und theoretisch orientierten Beiträgen aus Cultural Studies, Rhetorik und Literaturwissenschaft, die bewusster und/oder mit offenerem Blick zu importieren und in Bemühungen um internationale Fachgeschichtsschreibung zu integrieren (so Irmela Schneider auf dem Podium, ähnlich Schüttpelz) die Abstraktionsleistungen lokaler Theorieproduktion keineswegs schmälern müsste.

Worum es geht, sind - abschließend - letztlich eben theoretische Verhandlungen zur Sache. Gegen die genannten Hegemonialmotive deutscher Kulturwissenschaft, deren Charakterisierung im Einklang mit dem hier Gesagten lediglich als gezielt fokussierte Momentaufnahme zu verstehen ist, würde ich selbst in der Tat eine kräftige Dosis jener «weichen Mittelchen [sic] verschreiben, die der (in der Debatte ebenfalls wiederholt zitierten) aktuellen Krise deutscher Medienwissenschaft vielleicht qua Balance effektiver entgegenwirken könnten als hharte> Totalisierungstheoreme: software, Medienästhetik, Rhetorik, in Verbindung mit Akzenten auf historischer Differenz, synchroner Vielstimmigkeit, einer bei Foucault geborgten anti-monarchischen Machtkonzeption und einem Interesse an Handlungsspielräumen diesseits von Souveränitätsphantasmen. Das ist prinzipiell alles andere als neu, aber vielleicht im Namen emphatisch transnationaler Theorieproduktion gegen die Sehnsüchte nach eindeutig-nationalen Referenzen - und mit Lust an der fortgesetzten Debatte im Abstrakten wie Konkreten - weiterhin sehr produktiv. 
1 Jürgen Kocka, German History before Hitler: The Debate about the German Sonderweg, in: Journal of Contemporary History 23, 1988, 3-16.

2 Vgl. z. B. Forum Transnationalism, in: H-German, dort datiert Januar 2006, http://www.h-net.org/ german/discuss/Trans/forum_trans_index.htm, gesehen am 1.5.2009.

3 Benedict Anderson, Imagined Communities: Reflections on the Origin and Spread of Nationalism, rev. ed. London (Verso) 1991 (vgl. Winthrop-Young, Von gelobten und verfluchten Medienländern. Kanadischer Gesprächsvorschlag zu einem deutschen Theoriephänomen, in: Zeitschrift für Kulturwissenschaften, 2/2008, S.113-127, 124).

4 Vgl. z. B. Fatima El Tayeb, Dangerous Liaisons: Race, Nation and German Identity, in: Patricia Mazón, Reinhild Steingröver (Hg.), Not So Plain as Black and White. Afro-German Culture and History, 1890-2000, Rochester (University of Rochester Press) 2005, 27-6o.

5 Campe akzentuiert die strukturelle Skandalposition, die der Technik im großen System der Philosophie zukommt und Lethen u. a. die vorherrschende Abwendung von der sozialen Sphäre des Austauschs zwischen extremen Positionen als signifikante Akzente deutscher Theoriebildung.

6 Die von Gumbrecht ergänzend genannten institutionellen Komponenten finde ich weniger einleuchtend, mit Ausnahme der Stilisierung des (idealtypisch männlichen) deutschen Professors zur Figur der Wahrheitsverkündung, d. h. seiner im Vergleich z. B. zu den USA noch größeren sozialen Autorität in Verbindung mit dem traditionell personalisierten, mittlerweile immerhin partiell reformierten persönlichen Abhängigkeitssystem universitärer Personalstruktur, das ich selbst (ähnlich wie Winthrop-Young, S. 120) polemisch als feudal beschreiben würde. Dagegen hilft, wie im Hinblick auf Balkes Replik zu ergänzen ist, selbstredend kein universitärer Absolutismus (S. 131), sondern nur - mit Bitte um Entschuldigung für die historische Plattitüde - mehr Demokratie.

7 Antworten von Florian Cramer, Medienwissenschaft: Ein deutscher Sonderweg?, dort datiert 19.4.2009, http:/|medienumbrueche.uni-siegen.de/ groups/medienwissenschaften/blog/, gesehen am 27.5.2009.

8 Sybille Krämer, Medium, Bote, Übertragung: Kleine Metaphysik der Medialität, Frankfurt/M. (Suhrkamp) 2008, 10.

9 Leise Zweifel habe ich allerdings hinsichtlich einiger Adepten in der anschließenden Online-Debatte-vgl. z. B. Peter C. Krells dezidiertes Plädoyer für Isolation, in: 3 Responses to «German Media Theory: Too shy to admit its own greatness", dort datiert 14.4.2009, http://netzmedium. de/2009/04/10/german-media-theory-too-shy-to-admit-its-own-greatness/, gesehen am 29.5.2009.

\section{Literatur:}

«Medienwissenschaft. Eine transatlantische Kontroverse» (Debattenteil der Zeitschrift für Kulturwissenschaften, 2/2008, Tübingen (transcript), II3-I52, mit einem Statement und einem Schlusswort von Geoffrey Winthrop-Young sowie Repliken von Friedrich Balke, Rüdiger Campe, Helmut Lethen und Ludwig Pfeiffer)

\section{Webvideos:}

«Medienwissenschaft: Ein deutscher Sonderweg?» Podiumsdiskussion an der Universität Siegen am 22.4. 2009 (mit einem Eröffnungsreferat von Hans Ulrich Gumbrecht sowie Friedrich Kittler, Irmela Schneider, Hartmut Winkler, Geert Lovink und Erhard Schüttpelz; im Netz dokumentiert unter: $\underline{h t t p: / / w w w . ~}$ fk615.uni-siegen.de/de/media.php).

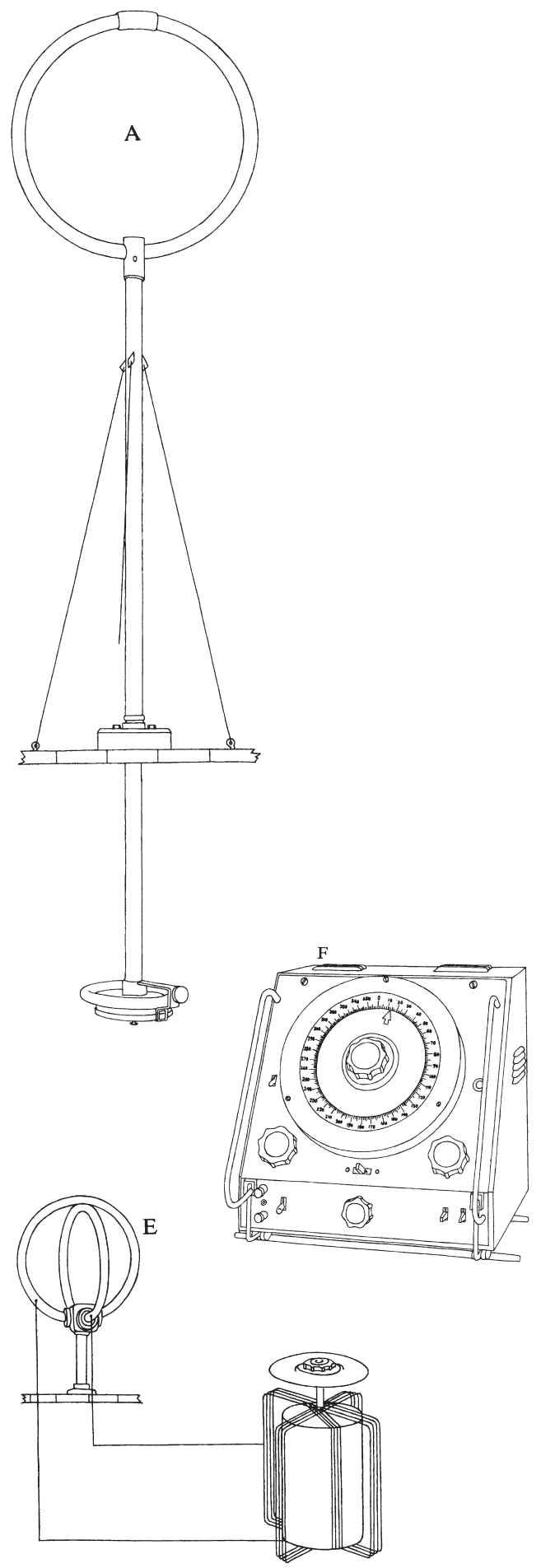

